\title{
Diacronie
}

Studi di Storia Contemporanea

$N^{\circ} 8,4 \mid 2011$

Le sembianze di Clio: approcci alla storia

\section{Internet Archive e nuove tipologie di fonti storiche}

\section{Slobodan Mandić}

Traduttore: Laura Uremović e Jasmina Mihajlović

\section{(2) OpenEdition}

\section{Journals}

\section{Edizione digitale}

URL: http://journals.openedition.org/diacronie/3561

DOI: 10.4000/diacronie.3561

ISSN: 2038-0925

\section{Editore}

Association culturelle Diacronie

\section{Notizia bibliografica digitale}

Slobodan Mandić, «Internet Archive e nuove tipologie di fonti storiche », Diacronie [Online], N ${ }^{\circ}$ 4 | 2011, documento 7, Messo online il 29 octobre 2011, consultato il 19 avril 2019. URL : http:// journals.openedition.org/diacronie/3561 ; DOI : 10.4000/diacronie.3561 


\title{
Diacronie
}

\section{Internet Archive e nuove tipologie di fonti storiche}

\author{
Slobodan MANDIĆ * \\ traduzione di Laura UREMOVIĆ, Jasmina MIHAJLOVIĆ *
}

Lo studio si occupa della specificità delle nuove tipologie di fonti storiche di tipo elettronico (pagine web, collezioni digitalizzate dei documenti, posta elettronica, blog, database ecc.) $e$ delle sfide che esse pongono alla storiografia e all'archivistica contemporanea. Il funzionamento e l'importanza di questo tipo di archiviazione vengono approfonditi analizzando il modello dell'Internet Archive che, grazie alla piattaforma del programma Wayback Machine e a circa 150 miliardi di pagine Web archiviate, rappresenta il più grande archivio elettronico al mondo.

I l concetto di fonte comprende tutti gli aspetti della vita umana. Tutte le tracce dell'attività umana sono considerabili come "fonti" se utili a ricostruire determinati periodi del processo storico. Se la ricostruzione storica deve fondarsi su basi il più possibile attendibili, dobbiamo sapere quali testimonianze del passato esistano e dove si trovino'. Le fonti storiche si possono trovare in situ, nel luogo del ritrovamento, ma per poterle conservare ed elaborare, vengono trasferite in appositi spazi: musei, biblioteche, archivi. Gli archivi rappresentano, come sottolinea Knut Kjeldstadli: «la nostra memoria collettiva»². Il compito di ogni archivio è proteggere il patrimonio culturale, che conserva e assicura alle presenti e future generazioni la conoscenza dei fatti del passato. Lo svolgimento delle due funzioni (preservazione e consultabilità del materiale) richiede diverse modalità di reperibilità del posseduto del sistema archivistico affinchè la fruizione di tale contenuto venga assicurato tramite l'uso delle forme alternative esistenti e l'utilizzo

\footnotetext{
${ }^{1}$ GROSS, Mirjana, Historijska znanost. Razvoj, oblik, smjerovi [La scienza storica. Sviluppo, forma, tendenze], Zagabria, SNL, 1980, pp. 232-236.

${ }^{2}$ KJELDSTADLI, Knut, Prošlost nije više što je nekad bila. Uvod u istoriografiju [Il passato non è più quello che era. Introduzione alla storiografia], Belgrado, Geopoetica , 2004, p. 69.
} 
degli originali venga ridotto il più possibile. Le possibili "copie", le forme alternative di utilizzo del sistema archivistico, sono: fotocopia, microfilm e documento digitale3.

Esiste un numero infinito di indirizzi Internet in cui è possibile trovare materiale: dalle fonti storiche scritte, quali corrispondenza privata, diari, memoriali, autobiografie, leggi, contratti, relazioni, al materiale audiovisivo che comprende quadri, fotografie, manifesti, mappe, stemmi, audioscritture, film ecc. ${ }^{4}$ Un grande vantaggio offerto da Internet per quanto riguarda le fonti storiche, è costituito dalla possibilità di evitare uno dei problemi principali legati alla rappresentatività del materiale di cui lo storico dispone. Nel caso in cui non esistano fonti riferite ad un determinato ambito geografico e ad un periodo storico, i risultati della ricerca non possono essere generalizzati e vanno limitati esclusivamente al contesto e al periodo a cui si riferiscono le fonti ${ }^{5}$. I servizi Internet permettono tuttavia ai ricercatori di allargare notevolmente i propri orizzonti di ricerca, offrendo la possibilità di rintracciare più facilmente i fondi documentari di interesse e di ottenere informazioni di particolare interesse. Inoltre, la decentralizzazione portata da Internet e la possibilità offerte dalla pubblicazione di materiale da parte di una cerchia allargata comprendente istituzioni e singoli, permettono un allargamento della rappresentatività del corpus documentario sia quantitativo (per esempio la consultazione degli archivi tramite le presentazioni internet delle istituzioni archivistiche), sia qualitativo (ad esempio le collezioni private di fotografie, manifesti, audioscritture, diari di persone comuni ecc.). Così come in passato la realizzazione di microfilm di documenti permetteva allo storico di evadere dalla ricerca storica tradizionale, evitandogli di passare ore in archivio a trascrivere pazientemente ogni singolo documento o parti di esso $^{6}$, allo stesso modo oggi lo storico ha a disposizione Internet, database elettronici, posta elettronica, CD-ROM, macchine fotografiche digitali...

Le fonti storiche primarie, o le fonti di primo ordine, sono le tracce del passato che derivano direttamente da un fatto storico, a differenza di quelle che trattano lo stesso fenomeno storico tramite un intermediario. Secondo Roger Griffin, è proprio nella ricerca di fonti primarie che Internet sta effettivamente manifestando tutto il suo

3 PORUBOVIĆ-VIDOVIĆ, Olivera, «Digitalizacija arhivske građe kao alternativa korišcenju originala», in Arhivska građa kao izvor za istoriju. Međunarodni naučni skup 15-16 maj 2000. god., [La digitalizzazione del sistema archivistico come alternativa all'uso degli originali in Il sistema archivistico come fonte per la storia. Convegno scientifico internazionale 15-16 maggio 2000], Belgrado, Arhiv Srbije, 2000, p. 292.

4 Sulle fonti storiche elettroniche e sulla loro catalogazione cfr. MANDIĆ, Slobodan, Kompjuterizacija $i$ istoriografija 1995-2005 [Computerizzazione e storiografia 1995-2005], Belgrado, Istorijski arhiv Beograda, 2008, pp. 48-68.

5 GROSS, Mirjana, Historijska znanost., cit., p. 273.

${ }^{6}$ Ibidem, p. 252. 
"splendore"7. Oltre ai siti Web creati dai dipartimenti universitari di storia, dai gruppi internazionali di storici, archivisti, archivi, musei, esiste un numero elevato di pubblicazioni online, create da storici e archivisti non professionisti così come da singoli in possesso di fotografie, cartoline, lettere e di tutto ciò che è legato ad un determinato periodo o fatto storico che si pensa possa essere d'interesse per altre persone. Gran parte di questo materiale viene trascurato dagli editori in quanto non è abbastanza commerciabile da poter coprire le spese di pubblicazione. ${ }^{8}$

Come fonti primarie possono essere utilizzati anche documenti che sono essi stessi prodotto dell'era informatica9. In un epoca in cui le tecnologie d'informazione hanno sostituito un grande numero di forme tradizionali di mediazione tra le persone, in cui la comunicazione avviene tramite posta elettronica o gruppi di discussione, i ricercatori hanno la possibilità di attingere anche a questo tipo di materiale nel loro lavoro. Possono, ad esempio, risultare di grande interesse i forum elettronici che riuniscono i testimoni di un fatto storico, come i veterani della Seconda guerra mondiale ${ }^{10}$, oppure, nel caso in cui una persona decida di ricostruire il proprio passato nell'ultimo decennio, un grande aiuto potrebbe essere fornito dall'archivio della corrispondenza elettronica personale. Fino a poco tempo fa, le testimonianze individuali su determinati fatti storici erano disponibili esclusivamente ad una cerchia ristretta di persone che frequentavano gli archivi e i centri di documentazione, ma, grazie all'utilizzo di Internet e delle nuove tecnologie, oggi la cerchia di utenti si sta allargando notevolmente.

Allo stesso modo una fonte storica d'interesse, soprattutto per coloro che si occupano di storia contemporanea, può essere rappresentata dai blog, ossia diari in forma elettronica pubblicati online, le cui popolarità e diffusione testimoniano

\footnotetext{
7 GRIFFIN, Roger. A., «Finding Primary Sources on the Internet», in Using the Internet as a resource for historical research and writing, Austin 1999,

URL: <http://www.austincc.edu/history/inreso4prm.html> [consultato il 25 novembre 2005]. ${ }^{8}$ STRADLING, Robert, Nastava evropske istorije dvadesetog veka [L'insegnamento della storia del ventesimo secolo], Belgrado, Ministarstvo prosvete i sporta Republike Srbije, 2003, pp. 115116.

9 Secondo Le Coadic «il documento è il concetto generico che definisce gli oggetti come portatori d'informazioni. Il documento è nella sua totalità l'artefatto che rappresenta o esprime, con l'aiuto di segni grafici o iconografici (parole, quadri, diagrammi, mappe, figure, simboli), con l'aiuto di segni audio e video (scritti cartacei o elettronici), un oggetto, un'idea o un'informazione, viene considerato un documento cartaceo o eletronico a seconda della modalità di trasmissione», Le COADIC, Yves-Fransois, Nauka o informacijama, [La scienza dell'informazione], Belgrado, Clio, 2005, p. 9.

${ }^{10}$ Un ottimo esempio di tale presentazione è rappresentato dal sito del progetto European Resistance Archive che ha come scopo principale la conservazione delle testimonianze individuali dei singoli che hanno partecipato ai movimenti di resistenza in Europa durante la Seconda guerra mondiale. Nella presentazione Internet dell'Archivio sono disponibili le interviste in formato video e testo, ed ogni testimonianza è seguita dalla biografia della persona intervistata e da una piccola galleria di documenti. European Resistance Archive, URL: <http://www.resistance-archive.org> [consultato il 24 settembre 2009].
} 
un'evoluzione di carattere globale legata alla generale computerizzazione della società. Un esempio che illustra la comprensione di questo fenomeno e la sua influenza sulla storia della quotidianità, è stato il progetto One Day in History, promosso sul portale della Biblioteca nazionale britannica: tutti i cittadini sono stati invitati a scrivere un blog personale, ossia un diario, in risposta alla domanda "Cosa fate il 17 ottobre 2006?”. In questo modo la Biblioteca nazionale britannica ha tentato di scattare un'istantanea storica della vita quotidiana della nazione: i cittadini descrivendo da soli la propria vita hanno permesso alla biblioteca di archiviare una sorta di diario nazionale per le future generazioni ${ }^{11}$.

In alcuni casi sono gli archivisti stessi a scrivere blog che offrono a tutti gli interessati importanti informazioni sulle loro attività professionali. Sul portale archivistico dell'UNESCO, così come sul portale dell'ArchivesBlogs, è possibile trovare decine di link a blog dedicati all'archivistica in diverse lingue ${ }^{12}$. In questo modo, oltre ad offrire informazioni tecniche, si costruisce una comunità specifica di archivistinetizen.

Il passaggio dalle forme tradizionali alla forma elettronica di espressione e alla comunicazione tramite le tecnologie d'informazione e comunicazione contemporanee, sta gradualemente portando alla scomparsa delle vecchie tipologie di fonti storiche: lettere, cartoline, diari, telegrammi, scritti; proprio come l'invenzione della stampa segnò un grande cambiamento per la civiltà agli inizi dell'età moderna. Da un lato queste fonti storiche diventano sempre più arcaiche e meno utilizzabili, dall'altro si arriva alla comparsa di rispettivi "cloni” in forma elettronica: siti Web, e-mail, forum di discussione, liste di servizi, blog e simili. Questi possono essere considerati come nuove tipologie di fonti storiche, sia per la specificità della forma elettronica, sia per i frequenti cambiamenti del contenuto delle pagine e per la necessità di creare strumenti specifici destinati alla loro critica, ad esempio per quanto riguarda la determinazione dell'autenticità ${ }^{13}$.

${ }^{11}$ BROWN, Miland, «One Day in History - History Matters», 17 ottobre 2006, in World History Blog,

URL: $\quad$ http://world-history-blog.blogspot.com/2006/10/one-day-in-history-historymatters.html $>$ [consultato il 24 ottobre 2006].

12 UNESCO Archives Portal, Mailing Lists and Blogs,

URL: <http://www.unesco-ci.org/cgi-bin/portals/archives/page.cgi?d=1\&g=91> [ consultato il 25 novembre 2009];

ArchivesBlogs - a syndicated collection of blogs by and for archivists,

URL: <http://archivesblogs.com/> [consultato il 25 novembre 2009].

${ }_{13}$ FOSTIKOV, Aleksandra, MANDIĆ, Slobodan, «New categories of historical sources: e-mail and forum. Internet communacation and history», in Pregled Nacionalnog centra za digitalizaciju, n. 10, 2007, p. 41. 
Un'altra significativa problematicità legata a queste nuove tipologie è rappresentata dalla loro classificazione, in particolare dalla divisione delle fonti tra private e pubbliche, complicata dalla tendenza ad eliminare le frontiere tra lo spazio pubblico e privato nella vita dell'uomo contemporaneo. L'esempio più evidente è il già menzionato fenomeno 'blog', il diario elettronico, la cui pubblicazione online è particolarmente semplice - non richiede l'uso di nessun tipo di software complicato - e che milioni di persone hanno già utilizzato per ritagliarsi uno spazio cibernetico personale.

Le tendenze attuali portano a nuove sfide e forme di collaborazione, da un lato tra storici, archivisti, bibliotecari, dall'altro tra ricercatori nel campo delle scienze dell'informazione e della matematica. La lenta scomparsa delle tracce della comunicazione scritta (lettere, cartoline, diari) influisce anche sullo studio della storia contemporanea, così come pone nuove sfide all'archivistica e alla metodologia delle ricerche storiche. Fino a poco tempo fa tale documentazione scritta permetteva lo studio sia della storia politica sia della vita privata del singolo. Oggi, tuttavia, questi tipi di documenti stanno scomparendo. Per chi studia la storia contemporanea, tale evidenza rappresenta un problema particolare per quanto riguarda la raccolta e l'uso delle fonti. I futuri storici, i ricercatori di qualsiasi tematica cronologicamente legata all'era della (post)informazione, saranno privi di un importante gruppo di fonti, finora conservate in archivi, in biblioteche e in altre istituzioni. La comparsa di nuove tipologie di fonti può parzialmente compensare la scomparsa di quelle vecchie. Tuttavia, una tale sostituzione richiede un ulteriore perfezionamento dell'archivistica contemporanea e della metodologia storica. Come già menzionato, il materiale reperito su Internet, poi masterizzato su CD-ROM o supporti simili, può avere un suo equivalente in forma stampata, che può essere identico, simile, o completamente diverso dalla forma elettronica del documento. Le fonti elettroniche comprendono, in senso stretto, i documenti esistenti esclusivamente in forma elettronica. Generalmente, si possono suddividere in due gruppi principali:

Web e fonti online: pagine World Wide Web, siti FTP, comunicazione sincronizzata online (chat), blog, e-mail private, e-mail dalla lista dei servizi, forum, gruppo di discussione, commenti pubblici

2. Materiale documentario archiviato o fonti elettroniche scritte in varie modalità di trasmissione (CD-ROM, DVD, hard disk, floppy, nastri magnetici ecc.): collezioni archiviate di documenti, libri, riviste, microfilm, e simili; 
materiale audiovisivo (fotografie, film, audioscritture); database; presentazioni multimediali

Esistono numerosi problemi legati alla presentazione, all'utilizzo e all'applicazione delle fonti elettroniche. La conseguenza più importante che appare nella presentazione dei contenuti in varie tipologie di formato elettronico, in particolare in HTML (quando l'intero contenuto di un documento, indipendentemente dalla sua portata, è di una sola pagina) riguarda il problema della citazione. Su Internet si possono trovare tante cosiddette "Guide alle citazioni", ed esistono anche guide specializzate per singoli settori scientifici, così come per la storiografia ${ }^{14}$. Un problema particolare consiste nel trovare il modo di riordinare i dati recuperati dal Web (ad esempio, per necessità di controllo) a causa di aggiornamenti periodici, cambio d'indirizzo, scomparsa del sito e altri simili inconvenienti. Riguardo l'attendibilità delle informazioni trovate su Internet, circa la loro verifica, è necessario sottolineare che le informazioni elettroniche trovate nello spazio cibernetico non dovrebbero scomparire per sempre. Per recuperare tali informazioni, non rintracciabili su indirizzi precedenti, è utile fruire dei motori di ricerca, grazie ai quali è spesso possibile individuare il nuovo indirizzo URL per il documento scomparso, non più rinvenibile nella pagina dove lo avevamo trovato in precedenza.

Un altro modo per cercare informazioni e siti su Internet, ai quali, a prima vista, non sembra più possibile accedere, è utilizzare Internet Archive.

Internet Archive ${ }^{15}$ è stato istituito come organizzazione non profit a San Francisco nel 1996 sotto la supervisione di Brewster Kahle, esperto in intelligenza artificiale e biblioteche digitali. L'intenzione principale era quella di archiviare l'intero contenuto di Internet e delle risorse multimediali in formato digitale. Questa eccellente idea è stata implementata con successo: alla fine del 2005 erano già stati archiviati più di 40 miliardi di pagine Web, mentre si è arrivati a 150 miliardi nel 2009. Internet Archive, grazie all'utilizzo della piattaforma del programma Wayback Machine, crea "registrazioni" di pagine World Wide Web, software, film, testi, musica ed audioscritture, che successivamente salva in un database protetto. Per garantire la protezione e la conservazione delle registrazioni, tutti i dati memorizzati nell'Archive

${ }^{14}$ CROUSE, Maurice, Citing Electronic Information in History Papers, 4 April 2009,

URL: <http://history.memphis.edu/mcrouse/elcite.html> [consultato il 27 novembre 2009]. Sulla questione della citazione delle fonti elettroniche vedere anche: MANDIĆ, Slobodan, «Problem citiranja elektronskih informacija» [Il problema della citazione delle informazioni elettroniche], in Tokovi istorije, n. 1-2, 2007, pp. 221-232.

${ }^{15}$ Per un approfondimento su Internet Archive cfr. Internet Archive: About IA, URL: <http://www.archive.org/about/about.php> [consultato il 26 novembre 2009]. 
sono salvati anche nella Biblioteca di Alessandria e ad Amsterdam. Tutte le collezioni conservate nell'archivio Internet sono disponibili a titolo gratuito.

Nella pagina Web di Internet Archive è possibile inserire nel campo di ricerca l'indirizzo URL desiderato $\mathrm{e}$ in questo modo ottenere un prospetto ordinato cronologicamente dei cambiamenti registrati avvenuti in un sito ${ }^{16}$. Le pagine risultano visibili agli utenti per un periodo che va dai sei ai dodici mesi successivi all'archiviazione, mentre gli utenti che desiderano archiviare in modo permanente determinati dati ed incominciare subito ad utilizzarli, hanno la possibilità di creare, a pagamento, un accesso al sistema Archive-It offerto da Internet Archive. Inoltre, è stato creato un motore di ricerca avanzato grazie al quale si possono ricercare fonti digitali a seconda del tipo di modalità di trasmissione (immagini in movimento, testi, audioscritture, software, forum ecc.). In questo modo, non solo è possibile ritrovare informazioni considerate irrimediabilmente perse, ma anche seguire i cambiamenti avvenuti all'interno di determinate pagine Web. Con questo viene inoltre confermata la richiesta di indicare la data di accesso nel momento in cui vengono citate le informazioni prese da Internet. Sebbene, all'interno di Internet Archive, ognuno abbia il diritto di bloccare l'accesso alla propria presentazione, la quantità di dati conservata e le possibilità offerte agli utenti rappresentano uno strumento indispensabile per ogni ricercatore che si trovi ad utilizzare le fonti digitali nel proprio lavoro.

Prendendo in considerazione la decentralizzazione di Internet, l'accesso libero a gran parte delle informazioni e la dispersione di dati nei server e nei siti di tutto il mondo, Internet Archive rappresenta anche un possibile paradigma per progetti minori, specializzati o tematici, nell'archiviazione elettronica. Conseguentemente, si valuta la possibilità di archiviare contenuti Web e collezioni digitalizzate più ridotte, da realizzare grazie a software in grado di rispondere alle diverse necessità ed ai diversi scopi. Una delle possibili soluzioni potrebbe essere aspirare ad archiviare il patrimonio culturale digitalizzato di un popolo a partire dal materiale appartenente alle istituzioni interne allo Stato cui verrebbe poi aggiunto quello delle collezioni private, includendo anche il materiale disperso nei server di tutto il mondo, il cui accesso è completamente libero.

Lo sviluppo espansivo delle nuove tecnologie, l'infiltrazione di Internet in tutte le sfere dell'attività umana, gli archivi online, i database, i cataloghi delle biblioteche e i numerosi progetti di digitalizzazione delle fonti, così come le riviste scientifiche e i libri

${ }^{16}$ È possibile in questo modo accedere, ad esempio, alle presentazioni Internet registrate in Serbia ed usare Internet Archive come supporto e come fonte per la ricerca sulla storia dell'archivistica serba nell'ultimo decennio. Ciò risulta significativo di fronte al fatto che la maggior parte degli indirizzi Web è stata modificata a causa del cambio di dominio nel 2009. 
in formato elettronico, rappresentano una grande sfida per la comunità accademica, ma anche per lo sviluppo e la concezione della teoria e della pratica nell'archivistica contemporanea. Sebbene la nostra società diventi ogni giorno sempre più digitalizzata, e la quantità di materiale disponibile tramite diversi servizi Internet sempre più consistente, con connessioni sempre più veloci e maggiori possibilità di accesso, risulta evidente come la trasformazione delle strutture istituzionali non vada di pari passo con questo sviluppo. Affrontando il problema dell'archiviazione del materiale elettronico e avviando un progetto come Internet Archive, si apre una nuova dimensione nella concezione della conservazione e dell'utilizzo del patrimonio digitale, grazie alla quale le generazioni future non dovranno considerare il nostro presente come un periodo digitale buio. 


\section{* L'autore}

Slobodan Mandić ha conseguito la laurea presso il Dipartimento di Storia della Facoltà di Filosofia di Belgrado nel 2005. Attualmente lavora presso l'Archivio Storico di Belgrado come responsabile della Sezione per la gestione e l'utilizzo della documentazione. È autore del libroComputerizzazione e storiografia 1995-2005, pubblicato dall'Archivio storico di Belgrado (2008) e di altri contributi relativi a differenti aspetti dell'utilizzo delle nuove tecnologie in ambito storiografico e archivistico. Nel biennio 2007-2008 ha partecipato al progetto internazionale Women and Minority Documentation and Digital Presentation (Vienna, Blagoevgrad, Belgrado).

URL: < http://studistorici.com/progett/autori/\#Mandić >

\section{Per citare questo articolo:}

MANDIĆ, Slobodan, «Internet Archive e nuove tipologie di fonti storiche», Diacronie. Studi di Storia Contemporanea: Le sembianze di Clio: approcci alla storia, 29/10/2011,

URL:<http://www.studistorici.com/2011/10/29/mandic_numero_8/ >

Diacronie Studi di Storia Contemporanea $\beta$ www.diacronie.it

Risorsa digitale indipendente a carattere storiografico. Uscita trimestrale. redazione.diacronie@hotmail.it

Comitato di redazione: Marco Abram - Giampaolo Amodei - Jacopo Bassi - Luca Bufarale - Alessandro Cattunar - Alice De Rensis Barbara Galimberti - Deborah Paci - Fausto Pietrancosta - Martina Sanna - Matteo Tomasoni - Luca Zuccolo

Diritti: gli articoli di Diacronie. Studi di Storia Contemporanea sono pubblicati sotto licenza Creative Commons 2.5 . Possono essere riprodotti a patto di non modificarne i contenuti e di non usarli per fini commerciali. La citazione di estratti è comunque sempre autorizzata, nei limiti previsti dalla legge. 